Le développement durable sous le regard des sciences et de l'histoire : de la réflexion aux pratiques éducatives

Colloque des 12 et 13 octobre 2006 - Arras, France

Patrick Mardelle

\title{
OpenEdition
}

Journals

Édition électronique

URL : http://journals.openedition.org/ere/4112

DOI : $10.4000 /$ ere. 4112

ISSN : 2561-2271

Éditeur

Centr'ERE

Référence électronique

Patrick Mardelle, «Le développement durable sous le regard des sciences et de l'histoire : de la

réflexion aux pratiques éducatives », Éducation relative à l'environnement [En ligne], Volume 6 | 2007,

mis en ligne le 14 septembre 2007, consulté le 24 septembre 2020. URL : http://

journals.openedition.org/ere/4112; DOI : https://doi.org/10.4000/ere.4112 


\section{Le développement durable sous le regard des sciences et de l'histoire : de la réflexion aux pratiques éducatives}

Colloque des 12 et 13 octobre 2006 - Arras, France

\section{Patrick Mardelle}

\section{Le développement durable sous le regard des Sciences et de l'Histoire : \\ de la réflexion aux pratiques éducatives \\ et de formation}

12 et 13 Octobre 2006 - Arras

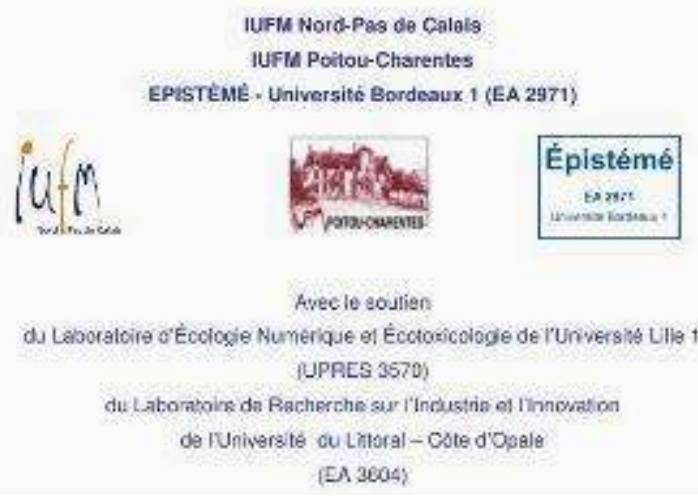

Ce colloque a été organisé par l'IUFM Nord-Pas-de-Calais, l'IUFM de Poitou-Charentes, le laboratoire EPISTEME de l'Université Bordeaux 1, et avec le soutien du Laboratoire d'Écologie Numérique et Écotoxicologie de l'Université Lille I et du Laboratoire de Recherche sur l'Industrie et l'Innovation. 
2 L'expression même de "développement durable ", comme chacun sait, ne fait pas consensus. Souvent présentée comme un oxymore, l'expression et son ambiguïté ont alimenté une partie des débats :

- recherche d'une définition qui ne soit pas trop large (donc confuse) à l'usage de ceux, enseignants ou éducateurs, qui auront pour rôle de l'expliciter au plus grand nombre;

- échange autour de la notion de développement au sens économique du terme et autour des notions de croissance, voire de consommation de masse qui lui sont souvent attachées ;

- réflexion autour du « développement humain » et autour de l'idée de décroissance.

3 La notion de «durabilité » n'est pas moins ambiguë. On parle même parfois de « l'enseignement de la durabilité ». La durabilité conduit à penser pour les générations à venir. Comment anticiper les besoins de générations que nous ne connaissons pas encore ? La réponse à l'objectif de durabilité ne conduit pas uniquement à des choix écologiques. Par exemple, les remèdes à la raréfaction des ressources naturelles peuvent passer par des solutions purement technologiques. Quelle que soit la définition, "parler de développement durable, c'est parler de pouvoir", d'une « logique de besoins contre une logique de profit». C'est un défi de plus pour le monde des enseignants et des éducateurs qui doit faire face à la difficulté de gérer cette dimension politique. En écologie comme en économie, il n'y a pas de lois «de bon sens » qui s'imposerait à tous et en toute circonstance. Il faut être conscient en particulier du risque qu'une certaine forme de néocolonialisme veuille imposer aux pays du Sud des règles de fonctionnement labellisées "développement durable » au nom d'une «bien pensance écologique », car si la notion de développement durable est une idée intégratrice, ses diagnostics et solutions qu'elle préconise ne sont pas universelles.

4 L'éducation à l'environnement vers un développement durable (EEDD), second axe fort du colloque, ne peut faire l'économie de jugements d'ordre existentiel: des modes d'existence qui se démarqueraient de la manière d'être "standard» de nos sociétés contemporaines (notions d'hésitation, de lucidité, de responsabilité et de capacité, d'abstraction humaine). "L'EEDD vient-elle bousculer la place des sciences dans la société ? Fait-elle reculer la vision positiviste de la science pour valoriser une vision constructiviste dans le cadre de l'interaction sociale?»

5 Les problématiques de l'EEDD imposent à l'enseignant d'aborder les problèmes d'échelle de temps et d'espace. L'échelle mondiale n'est pas pertinente. Par opposition la « ville durable » serait-elle la bonne échelle ? Si ce niveau est le bon pour travailler autour du sentiment d'appartenance, il ne recouvre pas une quelconque homogénéité sociale.

6 Une enquête a montré que 50 \% des enseignants n'ont pas de représentation concrète du développement durable. Il reste donc un très important travail d'explicitation. Dans cette perspective, le dialogue entre les sciences, bien que difficile, est plus que jamais nécessaire. Le développement durable est "sujet à interprétation, sans doute à récupération: il convient donc de se questionner sur les positions didactiques et épistémologiques, sur la formation des enseignants et sur les pratiques éducatives». "C'est une expertise citoyenne qu'il s'agit de construire, au moyen d'approches systémiques et actives (Sauvé, 1997) selon une modalité coopérative (Fortin-Debart et Girault, 2005) ». Dans ce contexte, si le premier vecteur de la mise en œuvre de l'EEDD est bien l'école, celle-ci doit également penser à s'ouvrir vers les acteurs de l'éducation informelle et le tissu associatif. 


\section{AUTEUR}

PATRICK MARDELLE

IUFM Poitou-Charentes 